\title{
Near-field characteristics of broad area diode lasers during catastrophic optical damage failure
}

\author{
Martin Hempel*a ${ }^{* a}$ Jens W. Tomm ${ }^{\mathrm{a}}$, Martina Baeumler ${ }^{\mathrm{b}}$, Helmer Konstanzer ${ }^{\mathrm{b}}$, Jayanta Mukherjee ${ }^{\mathrm{c}}$, \\ Thomas Elsaesser ${ }^{\mathrm{a}}$ \\ ${ }^{a}$ Max-Born-Institut, Max-Born-Strasse 2A, 12489 Berlin, Germany; \\ ${ }^{\mathrm{b}}$ Fraunhofer Institut für Angewandte Festkörperphysik, Tullastrasse 72, 79108 Freiburg, \\ Germany \\ ${ }^{c}$ Advanced Technology Institute, University of Surrey, Guildford, GU2 7XH, UK
}

\begin{abstract}
One of the failure mechanisms preventing diode lasers in reaching ultra high optical output powers is the catastrophic optical damage (COD). It is a sudden degradation mechanism which impairs the device functionality completely. COD is caused by a positive feedback loop of absorbing laser light and increasing temperature at a small portion of the active material, leading to a thermal runaway on a nanosecond timescale. We analyze commercial gain-guided $\mathrm{AlGaAs} / \mathrm{GaAs}$ quantum well broad area diode lasers in single pulse step tests. The near-field emission on the way to and at the COD is resolved on a picosecond time scale by a streak-camera combined with a microscope. In the final phase of the step tests the COD is occurring at $\sim 50$ times threshold current. The growth of the COD defect site is monitored and defect propagation velocities between 30 and $190 \mu \mathrm{m} / \mu \mathrm{s}$ are determined. The final shape of the damage is verified by opening the device and taking a micro-photoluminescence map of the active layer.
\end{abstract}

Keywords: high power broad area diode lasers, near-field, filamentation, degradation, catastrophic optical damage

\section{INTRODUCTION}

One of the reasons that limit the optical output power of today's semiconductor diode lasers is the catastrophic optical damage (COD). To prevent device failure via COD a deep understanding of the underlying physical mechanisms are necessary. An important issue on that way is to find a controlled way of performing COD test on devices. It was shown that the COD can be provoked by using high current short single pulses, eventually leading to the same result as seen in the case of continuous wave $(\mathrm{cw})$ operation. The advantage of using an elevated current is the significant reduction of the time from start of the life cycle of the device to the point where COD occurs from $\sim$ years to $\sim$ ns. This allows for triggering of experimental setups and therefore the usage of a wide variety of in-situ analytic methods.

When dealing with elevated output powers another laser property comes into focus of research - the optical near-field distribution (NF). Their properties and changes under extreme conditions are crucial for efficient coupling of these lasers to optical systems, particularly in the case where the diode lasers are used in pulse mode on an elevated current level. This operation regime is desired, e.g., in case of pumping solid state lasers. ${ }^{2}$

In this report we investigate the NF at increasing pump currents up to the point where COD starts. This current is the socalled COD threshold $\left(I_{\mathrm{COD}}\right)$. As shown earlier, a critical temperature $T_{\text {crit }}$ of $120^{\circ} \mathrm{C}-160^{\circ} \mathrm{C}$, see Ref. ${ }^{3}$, at the starting point of COD is necessary. After reaching this critical value a thermal runaway process starts, building up a positive feedback loop between increased temperature and absorption of laser light.

*hempel@mbi-berlin.de; phone +49(0)30/6392 1453; fax +49(0)30/6392 1459; www.mbi-berlin.de 


\section{EXPERIMENTAL}

The sample set consists of 20 commercial $808 \mathrm{~nm}$ edge emitting high-power broad area diode lasers. They are intentionally gain-guided with an $\mathrm{AlGaAs} / \mathrm{GaAs}$ quantum well $(\mathrm{QW})$. The devices are mounted $p$-side down and the laser stripes have a dimension of $50 \mu \mathrm{m} \times 1.4 \mathrm{~mm}$ (width $\times$ length). The front and rear facets have standard low reflective and high reflective coatings, respectively. The threshold currents of the lasers are $I_{\text {th }}=(0.21 \pm 0.01) \mathrm{A}$ and they are designed for a cw current of $(0.65 \pm 0.01)$ A with an optical output power of $(0.57 \pm 0.02) \mathrm{W}$. We will restrict this report to a few devices labeled A to E.

Figure 1 shows a scheme of the setup used. The diode laser is attached directly to a PicoLAS LDP-V 50-100 V3 pulse driver to ensure a short current rise time of $\sim 10 \mathrm{~ns}$. A thermo-electric cooler keeps the heat sink at a temperature of $(23.0 \pm 0.2)^{\circ} \mathrm{C}$. The NF is imaged by $25 \times$ microscope objective or in an updated experimental setup by a microscope with 50× objective to the horizontal entrance slit of a HAMAMATSU C1587 streak camera (equipped with the M1953 single sweep unit).

During the measurement of the devices we apply $300 \mathrm{~ns}$ long single pulses. For particular devices the pulse length is increased to $1000 \mathrm{~ns}$ to observe transient effects on this timescale. However, the measurements are starting from a current of $I_{0}=1.6 \mathrm{~A}$. This is significantly below the COD threshold for short pulses of these lasers. After taking a streak camera image and the transient of the current passing the laser, the current is increased by $0.5 \mathrm{~A}$ for the next single shoot. Each high current single shoot is followed by a low current one at $I_{0}$. This procedure - called step test - allows for determination of the lowest $I_{\mathrm{COD}}$ for each device and the detection of even small degradation signatures. For a more detailed description see Refs. ${ }^{1,4}$

The current in the step tests is increased up to $I_{\mathrm{COD}}$ where COD occurs. It is manifested in a region of decreased intensity in the NF which increases its width in time. Additionally, physical damage at the front facet is observed via an optical microscope. After the COD occurs it is possible to revive the COD defect growth process with another single pulse. The concept of $C O D$ re-ignition and its comparability to $\mathrm{cw}$ results is verified earlier. ${ }^{1}$

Some selected devices are opened after the described test by mechanically removing the $n$-contact and selective etching of the GaAs substrate and the buffer layer. ${ }^{5}$ Thereafter the QW plane is mapped by micro-photoluminescence $(\mu \mathrm{PL})$.

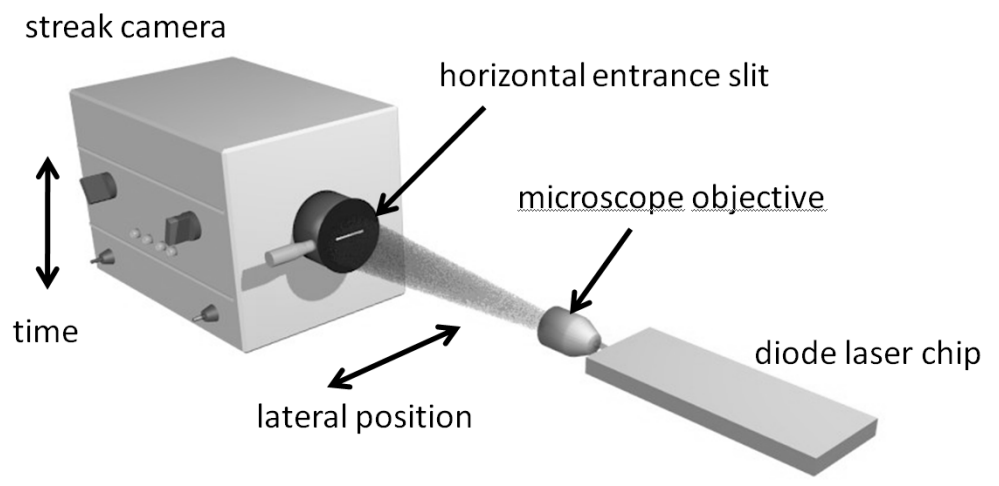

Figure 1. Scheme of the used experimental setup.

\section{RESULTS}

\subsection{Near-field at high operation currents}

Figure 2 shows the NF evolution during $300 \mathrm{~ns}$ long single pulses for device A, whose behavior is typical for the batch under investigation. The data for three different driving currents are shown, namely $I=6.0 \mathrm{~A}$ (representing approx. $29 \times$ the threshold current value), $7.5 \mathrm{~A}\left(36 \times I_{\mathrm{th}}\right)$, and $8.5 \mathrm{~A}\left(41 \times I_{\mathrm{th}}\right)$. The left column of Fig. 2 shows overviews over the entire $300 \mathrm{~ns}$ pulses with a temporal resolution of $\sim 680 \mathrm{ps}$. For reaching a higher resolution, images inside the pulses are taken on an enlarged time scale. These images are shown in the remaining columns of Fig. 2. Their temporal resolution is $\sim 18$ ps. The temporal position in the $300 \mathrm{~ns}$ pulse is indicated by the dashed lines and the letters in the overview images. The change of the NF pattern with time and current and the decreasing overall widths of the NF's are obvious. 
The latter mentioned effect is shown in detail in Fig. 3. In Fig. 3(a) the NF evolution during a $1 \mu$ s long pulse with a current of $I=4.5 \mathrm{~A}$ is shown for device B. Cuts along the lateral coordinate through this pulse are presented in Fig. 3(b) for three different delay times, indicated in (a) as dashed lines. In this particular example the intensity of laser emission from the left side (between 0 and $25 \mu \mathrm{m}$ ) is slightly weaker during the first $\sim 300 \mathrm{~ns}$ of the pulse. The area under the curves as shown in Fig. 3(b) is proportional to the optical output power of the laser. The values for these areas along the pulse of Fig. 3(a) are given in Fig. 3(c). The value stays almost constant over time as expected for the output power and observed for this type of device.

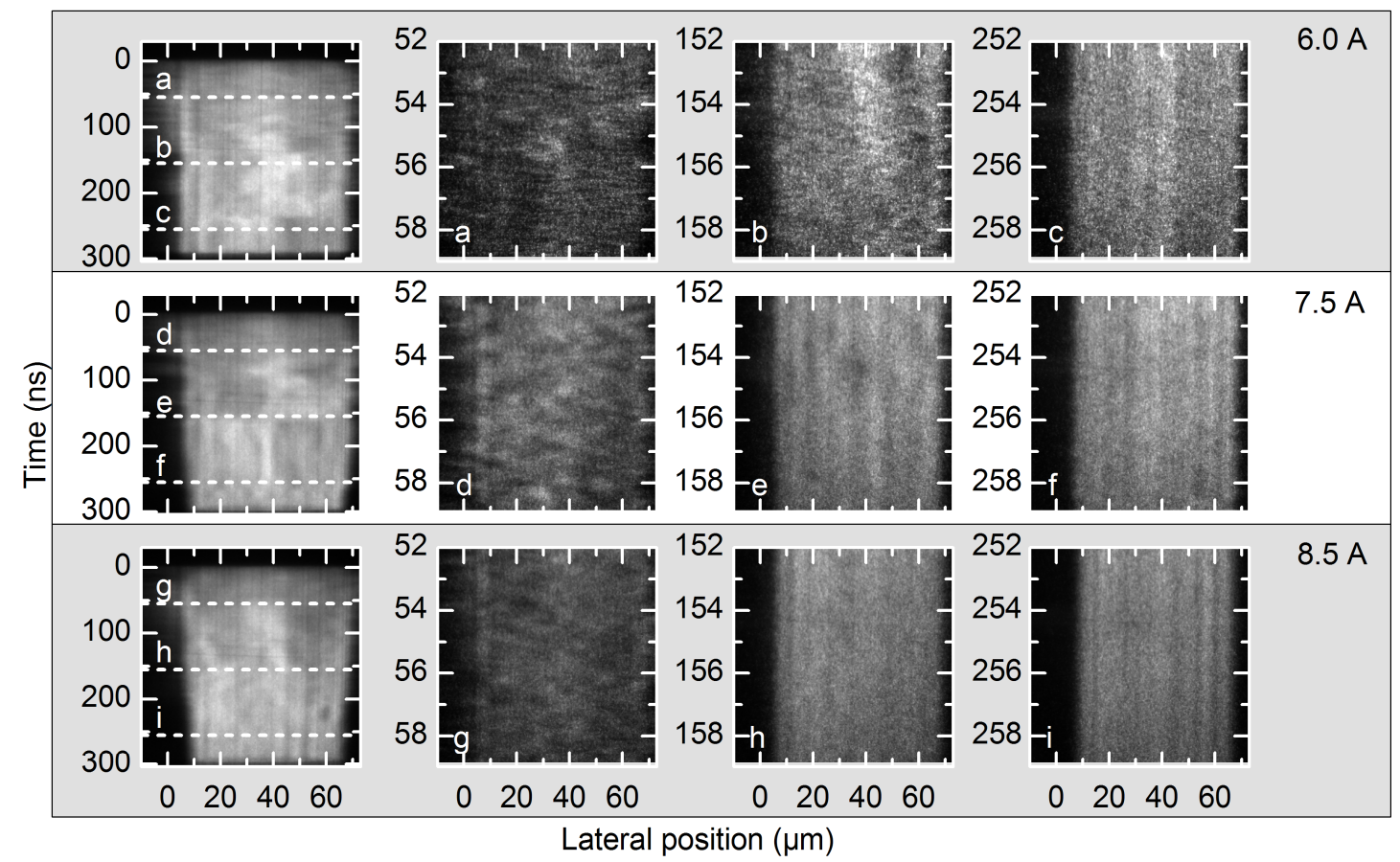

Figure 2. NF evolution of device A during a $300 \mathrm{~ns}$ long single pulse. The used currents are indicated. The left column gives overviews over the entire pulse, while the other images show the NF on an enlarged scale at the indicated positions.

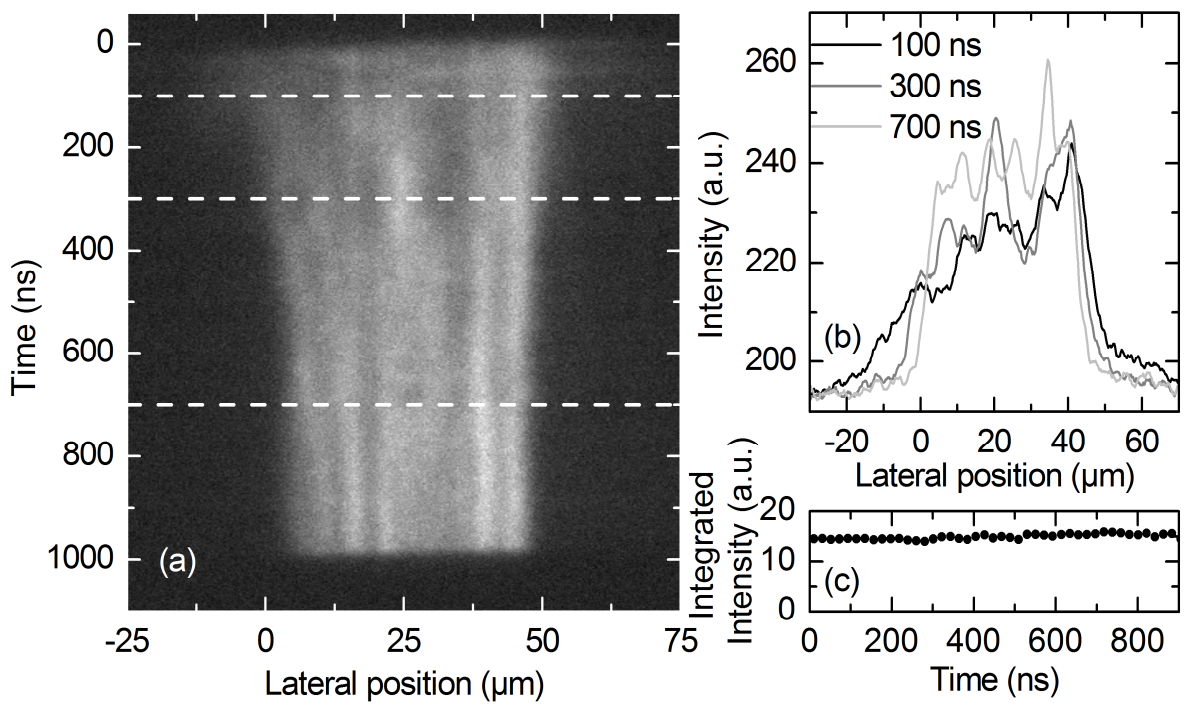

Figure 3. (a) NF evolution of a $1 \mu$ s long pulse of device B at $I=4.5 \mathrm{~A}$. (b) Cuts through the NF shown in (a) at the given times. The temporal position is additionally indicated in (a) by dashed lines. (c) Integrated area under curves as shown in (b). 


\subsection{Near-field en route to and during COD}

Following the step test concept presented in section 2, the COD threshold current is reached at a certain operation current. An example of the NF streak camera images obtained from a COD event is given in Fig. 4(a). Here the COD is provoked in device B at $I_{\mathrm{COD}}=7.5 \mathrm{~A}$ during a $1 \mu \mathrm{s}$ long single pulse. The start of COD related degradation, which manifests itself in an increasing region of reduced NF intensity, we call it NF gap, is indicated by an arrow (COD starts 930 ns after pulse begin). Figures 3(b) and (c) are showing the first and second re-ignition of the COD in subsequent pulses. The growth of the NF gap with ongoing time is clearly visible. Furthermore the lateral modes redistribute after the primary COD site occurs. This causes a raise of optical load at well separated locations.

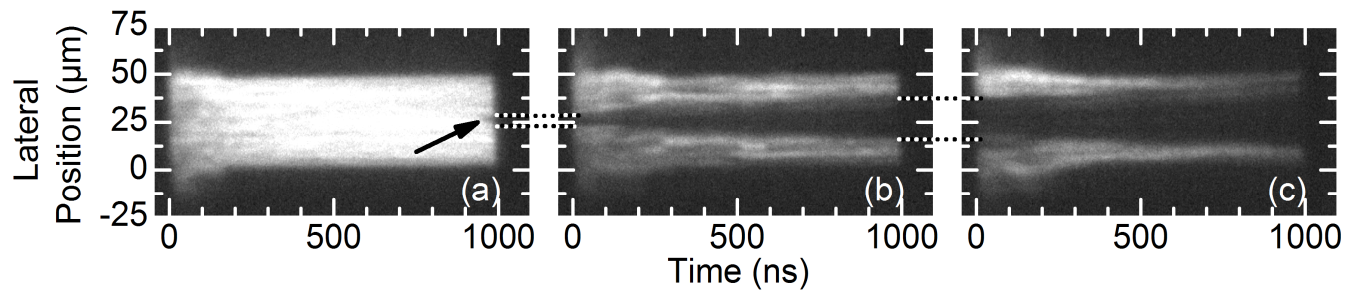

Figure 4. NF evolution in vicinity of COD for device B. (a)-(c) are showing three successive $1 \mu$ s long single pulses with $I=$

7.5 A. The first occurrence of COD is indicated by an arrow in (a). The further development of the NF gap is shown in (b) and (c).

After the step tests are finished some of the devices are opened and the QW plan is mapped by $\mu$ PL. Such an image is shown in Fig. 5(a). In this particular case the damage site of device $\mathrm{C}$ is shown, after experiencing a COD during a 300 ns long pulse of $I=10 \mathrm{~A}$ and the re-ignition of the process during the successive pulse with the same parameters. Altogether the time during damage growth is measured is $\sim 270 \mathrm{~ns}$. The position of the front facet is indicated in this image and also the maximal width of $11 \mu \mathrm{m}$ of the damage. Figure 5(b) gives a cut through the NF after the step test, before opening device $\mathrm{C}$, taken with an optical microscope at $I=0.5 \mathrm{~A}$, while sub-figure (c) gives a cut through the streak camera NF image at the trailing edge of the re-ignition pulse. Figure 5(d) gives an image of the front facet of device $\mathrm{C}$ after COD and COD re-ignition, taken with an optical microscope.

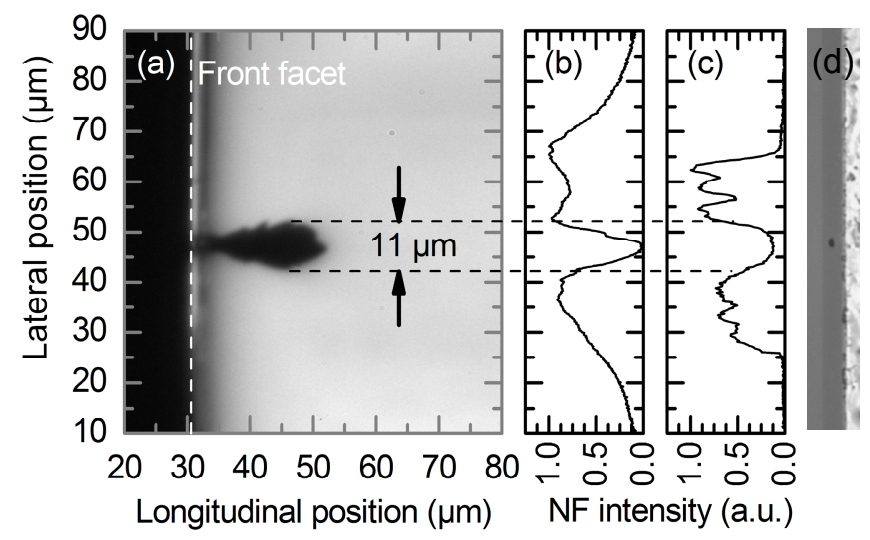

Figure 5. (a) $\mu \mathrm{PL}$ image of the QW plane of device $\mathrm{C}$, opened after COD and one COD re-ignition, both at $I=10 \mathrm{~A}$, giving a time of $\sim 270 \mathrm{~ns}$ for damage growth. (b) Cut through lasing NF at $I=0.5 \mathrm{~A}$, seen with optical microscope (after COD re-ignition, before device was opened). (c) Cut through lasing NF at the trailing edge in the streak camera image of COD re-ignition at $I=10 \mathrm{~A}$. The width of the internal damage of $11 \mu \mathrm{m}$ visible in (a) is indicated by the dashed lines in (b) and (c). (d) Image of the front facet taken with an optical microscope, lateral position scale is the same as in (a)-(c).

\section{DISCUSSION}

\subsection{Thermal behavior}

We start our discussion with the changes of the NF at higher pump currents up to slightly below $I_{\mathrm{COD}}$. The obvious reduction in the overall width of the NF is visible in Figs. 2, 3(a), and (b). The explanation of this effect is a rise in the active region temperature, which can be verified by the spectral shift of the laser emission. The results of such a 
measurement of device $\mathrm{D}$ is shown in Fig. 6. The points for $I=10 \mathrm{~A}$ are extrapolated from the $1.6 \mathrm{~A}, 6 \mathrm{~A}$, and 7 A data. This extrapolation to $10 \mathrm{~A}$ is done, because this current is the typical COD threshold value for the device batch under investigation, as will be discussed in detail later. The measured active region temperature rise leads, via the known $\mathrm{d} n / \mathrm{d} T$ relation $^{6}$, to a change of its refractive index of $\Delta n=6.5 \times 10^{-3}$ after $300 \mathrm{~ns}$ and $\Delta n=8.4 \times 10^{-3}$ after $1 \mu \mathrm{s}$, both at $I=10$ A. (The two mentioned points in time are of special interest, because these are typical pulse widths used for COD step tests.) This refractive index change causes a transition from the pure gain-guided device to a thermally-induced indexguided one, as theoretically shown in Ref. ${ }^{7}$ While the width of the NF is decreasing the optical output power remains constant, as shown in Fig. 3(c). This eventually increases the optical load at the facet, supporting light intensity triggered degradation mechanisms that start at the facet, like the COD.

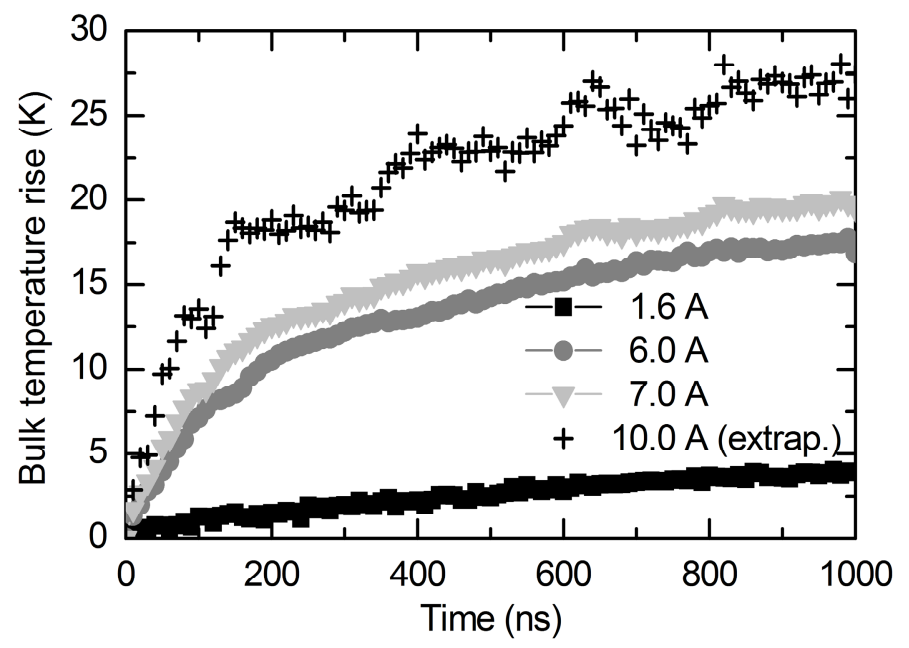

Figure 6. Bulk-temperature evolution for the investigated batch, shown data are measured for device D. The temperature rise is given with respect to the heat-sink temperature of $23^{\circ} \mathrm{C}$. The data for $I=10 \mathrm{~A}$ is extrapolated from the measured curves.

In addition to the decreasing width of the NF Fig. 2 shows fundamental changes in the spatio-temporal structure of it. For lower current and times closer to the leading edge of the pulse a clear zigzag motion of spots of higher intensities is visible. ${ }^{8}$ With the rise of the active region temperature the frequency of the motion of these main filaments increases and becomes eventually imperceptible according to the temporal resolution of the setup., ${ }^{90}$ For higher currents and times close to the trailing pulse edge a NF structure known from reports about thermal lensing effects in cw experiments is found. ${ }^{11}$

\subsection{COD kinetics}

The three successive 7.5 A single pulses in Fig. 4(a)-(c) are showing the time evolution of the growth of the NF gap. It is demonstrated that with short single pulses a time slicing of the COD related defect growth is possible. The damage growth is stopped by the pulse end and revived in the following pulse with the same current. The start-stop principle is also visible by comparing the widths of the NF gaps at the end of one pulse and at the beginning of the following one, as indicated by the dotted lines between (a) and (b) and (b) and (c). An advantage of the short pulses in the framework of the step test is that early damage stages can be prepared by stopping the test after the first COD sign is visible, i.e. after the pulse of Fig. 4(a) in this example. Furthermore, a wide variety of measurement techniques can be applied after each step, which cannot be used generally, e.g. due to restriction in measurements time, to investigate the fast COD kinetics.

In the following we will restrict the discussion to the devices $\mathrm{C}$ and $\mathrm{E}$, which are COD step tested with $300 \mathrm{~ns}$ long pulses. The main difference between these two devices is that $I_{\mathrm{COD}}=10 \mathrm{~A}$ for device $\mathrm{C}$, while $I_{\mathrm{COD}}=13 \mathrm{~A}$ for device $\mathrm{E}$. Taking a look at the distribution of COD threshold currents in the entire device batch, Fig. 7(a), device C represents a device at almost the middle of the distribution, while device E represents an extreme value on the higher current side. 

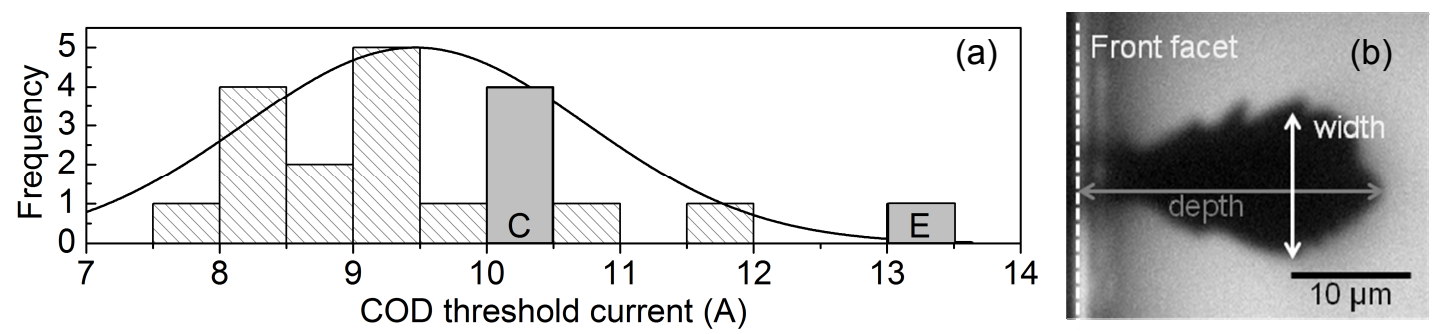

Figure 7. (a) Distribution of COD events for the investigated device batch with respect to the COD threshold current. The bins containing device $\mathrm{C}$ and $\mathrm{E}$ are denoted accordantly. (b) $\mu \mathrm{PL}$ map of the QW of device $\mathrm{C}$, the geometrical dimensions are labeled.

We take NF maps of the type of Fig. 4(a) from these devices and extract two values that will be compared afterwards:

- the width of the NF gap, Fig. 8(a), in case of COD and COD re-ignition of device C and COD of device E, and

- the reduction of the NF intensity in the middle of the NF gap, which is the starting site of COD defect growth, displayed in Fig. 8(b).

These two values can now be interpreted in terms of geometrical dimensions of the internal damage. The width of the NF gap seen in the streak camera image, Fig. 5(c), corresponds well to the internal damage of the defect, Fig. 5(a). Notice that the damage width visible at the facet, e.g. by inspection of the front facet with an optical microscope, is much smaller than this. The same width can be verified by a cut through the cw NF taken with an optical microscope, Fig. 5(b), at lower currents of $I=0.5$ A. By comparing the two cuts through the NF in Fig. 5(b) and (c) the above discussed active material heating due to different driving current can be ascertained. In this way the width of the NF gap gives a direct measure of the maximal width of the internal damage, which allows monitoring of the damage growth in that direction on a picoseconds timescale via our streak camera setup. The other value, the intensity at the COD site [Fig. 8(b)], is a measure for the longitudinal dimension, the depth along the resonator axis, of the damage area, also monitored in the same streak camera image. By having access to this geometrical information, see Fig. 7(b), during the damage formation we can compare the two devices.
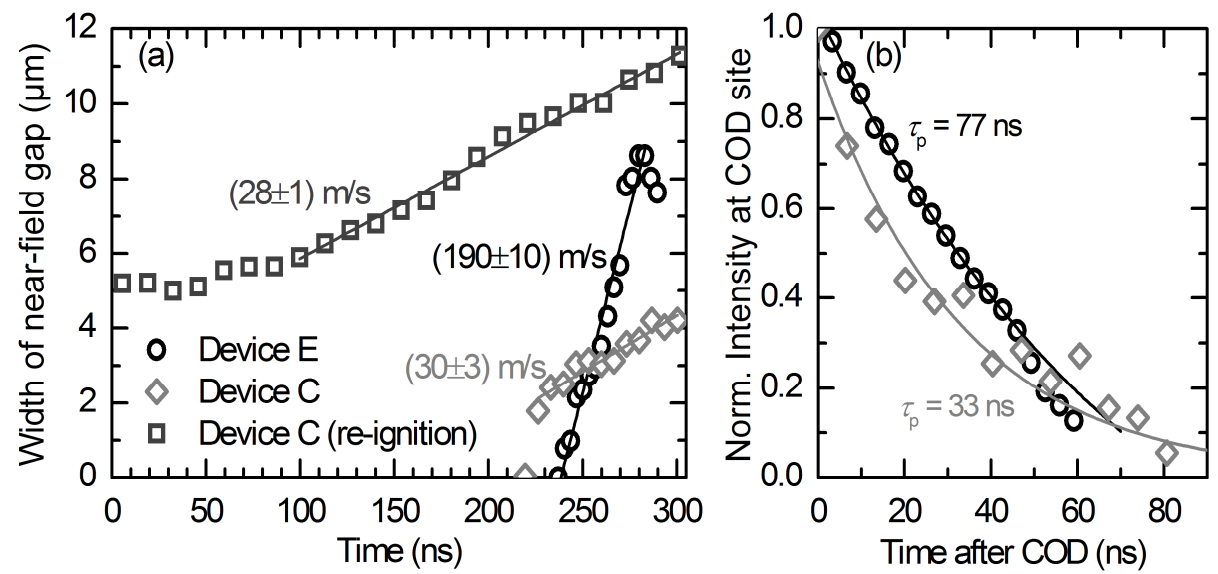

Figure 8. (a) Temporal evolution of the width of the NF gap during COD and, in case of device C, also during COD reignition. The solid lines indicate fits to the data and the growth velocities determined in this way are given next to the lines. (b) Comparison of the intensity drops after COD starts (zero point of the time axis in this plot is set to the $t_{\mathrm{COD}}$ values of the lasers). The curves are normalized to the value prior to COD onset. The solid lines indicate fits with exponential decay curves, the characteristic decay times obtained by these fits are given next to the data.

For both devices the starting point of damage creation lies between $220 \mathrm{~ns}$ and $240 \mathrm{~ns}$, Fig. 8(a). The degradation starts with the creation of a NF gap $\leq 2 \mu \mathrm{m}$ in less than $700 \mathrm{ps}$ and $\leq 1 \mu \mathrm{m}$ in $\leq 400 \mathrm{ps}$ in case of device $\mathrm{C}$ and $\mathrm{E}$, respectively. This jump like onset of damage growth represents most likely the thermal runaway ${ }^{3}$ as the fastest phase of COD. Thereafter the lateral expansion of the damage continues with constant velocities of (30 \pm 3$) \mathrm{m} / \mathrm{s}$ in case of device $C$ and 
$(190 \pm 10) \mathrm{m} / \mathrm{s}$ for device E. Device C have been subjected to another current pulse of same length and magnitude as used for COD ignition. The evolution of the damage width in this case of COD re-ignition is also shown in Fig. 8(a). The velocity here is $(28 \pm 1) \mathrm{m} / \mathrm{s}$ which is almost the same value as for the previous pulse, meaning that the energy feeding the growth process remains almost constant. In comparison the lateral damage expansion is faster in case of device E. In contrast the longitudinal damage growth is faster in case of device $\mathrm{C}$ up to $\sim 45 \mathrm{~ns}$ after COD starts, see characteristic decay times and slopes in Fig. 8(b).

The damage growth for device $\mathrm{C}$ is faster in longitudinal direction than for device $\mathrm{E}$, while the situation is vice versa in case of the lateral direction. This shows that the defect kinetics in the first $\sim 45 \mathrm{~ns}$ after COD starts is strongly affected by the COD threshold current.

\section{CONCLUSION}

In conclusion our experiments show the change from pure gain to thermally-induced index guiding for high pump levels even in single pulses of up to $1 \mu$ s pulse width. The measured higher optical load at the facet leads to an increased facet temperature and favors the occurrence of COD. Furthermore, the monitoring of the NF change with a streak camera setup is proven as a valuable tool to investigate the defect growth dynamics on a picoseconds timescale. Lateral damage growth velocities between $\sim 30 \mu \mathrm{m} / \mu \mathrm{s}$ and $\sim 200 \mu \mathrm{m} / \mu \mathrm{s}$ are found. An upper limit of $700 \mathrm{ps}$ for the thermal runaway

phase can be given. We have shown that the defect growth in lateral and longitudinal direction crucially depends on the COD threshold current of the particular device.

\section{REFERENCES}

[1] M. Hempel, F. L. Mattina, J. W. Tomm et al., "Defect evolution during catastrophic optical damage of diode lasers," Semiconductor Science and Technology, 26(7), 075020 (2011).

[2] R. L. Byer, “Diode-Laser - Pumped Solid-State Lasers," Science, 239(4841), $742-747$ (1988).

[3] J. W. Tomm, M. Ziegler, M. Hempel et al., "Mechanisms and fast kinetics of the catastrophic optical damage (COD) in GaAs-based diode lasers," Laser \& Photonics Reviews, 5(3), 422-441 (2011).

[4] M. Hempel, M. Ziegler, J. W. Tomm et al., "Time-resolved analysis of catastrophic optical damage in $975 \mathrm{~nm}$ emitting diode lasers," Applied Physics Letters, 96(25), 251105 (2010).

[5] M. Baeumler, J. L. Weyher, S. Muller et al., [Investigation of degraded laser diodes by chemical preparation and luminescence microscopy] Institute of Physics Conference Series, Templin, Germany(1998).

[6] D. E. Aspnes, S. M. Kelso, R. A. Logan et al., "Optical Properties of $\mathrm{Al}_{\mathrm{x}} \mathrm{Ga}_{1-\mathrm{x}} \mathrm{As}$," Journal of Applied Physics, 60(2), 754-767 (1986).

[7] J. Mukherjee, and J. G. McInerney, "Spatial mode dynamics in wide-aperture quantum-dot lasers," Physical Review A, 79(5), 053813 (2009).

[8] M. Hempel, J. W. Tomm, M. Baeumler et al., "Near-field dynamics of broad area diode laser at very high pump levels," AIP Advances, 1(4), 042148 1-6 (2011).

[9] I. Fischer, O. Hess, W. Elsasser et al., "Complex spatio-temporal dynamics in the near-field of a broad-area semiconductor laser," Europhysics Letters, 35(8), 579-584 (1996).

[10] H. Adachihara, O. Hess, E. Abraham et al., "Spatiotemporal chaos in broad-area semiconductor laser," J. Opt. Soc. Am. B, 10(4), 658-665 (1993).

[11]P. Crump, S. Böldicke, C. M. Schultz et al., "Experimental and theoretical analysis of the dominant lateral waveguiding mechanism in $975 \mathrm{~nm}$ high power broad area diode lasers," Semiconductor Science and Technology, 27(4), 045001 (2012). 